\title{
Evaluation of Dry Eye in patients with Psoriasis
}

\author{
Keywords: Psoriasis; Dry Eye Scoring System; Visual; Conjunctival \\ impression cytology

\begin{abstract}
Purpose: To evaluate the role of routine tear film tests such as Schirmer, tear film break up time (TBUT), Rose Bengal staining (RBS) and conjunctival impression cytology for dry eye evaluation in patients with
\end{abstract} \\ psoriasis.
}

Methods: A case control study was performed at two referral eye centers. Symptomatic patients with psoriasis were enrolled based on their response to (Dry Eye Scoring System, DESSC), a questionnaire of dry eye related symptoms (Table 1). They further underwent Schirmer, TBUT, RBS and conjunctival impression cytology $(\mathrm{CIC})$. Age and sex matched healthy subjects, served as controls. Means of groups were compared using t tests. Sensitivity and specificity of tear film tests for dry eye diagnosis was estimated by the area under receiver operating characteristic (ROC) curve. A correlation analysis (with regression) was done between dry eye symptoms and tear function tests in both groups, respectively. Adjusted Pearson's coefficient $\left(R^{2}\right)$ and slopes of normal probability plots were compared; $R^{2}>0.5$ was considered significant.

Results: The diagnostic accuracy of tear film tests was $\mathrm{CIC}$ $(A \cup C=0.969) \quad>$ TBUT $(A \cup C=0.962)>$ Schirmer $\quad(A \cup C=0.882)>R B S$ ( $A \cup C=0.880)$. Nelson grade $\left(R^{2}=0.840\right)$ correlated best with dry eye symptom severity. Schirmer $\left(R^{2}=0.612\right)$ and TBUT $\left(R^{2}=0.630\right)$ also correlated well but with a smaller magnitude. However, there was lack of correlation between symptom severity and $R B S\left(R^{2}=0.334\right)$.

Conclusion: Nelson grade correlates better with dry eye symptom severity as compared to TBUT, Schirmer and RBS. CIC is more sensitive and specific than routine tear function tests for dry eye evaluation in patients with psoriasis.

\section{Introduction}

Psoriasis is a chronic autoimmune disease that triggers skin to speed up its cellular growth cycle. In response to some antigenic stimuli, there is activation of plasmacytoid dendritic cells and immune cells; these cells in turn produce pro-inflammatory cytokines like interferon-alpha, interlukin-23 and interleukin-12 that activate $\mathrm{T}$-cells. Recruited $\mathrm{T}$ cells stimulate proliferation of keratinocytes [16].

Dryness of eyes is a common ophthalmic manifestation of psoriasis and may affect vision related quality of life. Recently, there have been significant inroads into pathophysiology of dry eye; inflammation of the ocular surface is now an integral part of disease process [7]. Dryness of eyes and the accompanying ocular surface inflammation may be associated with expression of pro-inflammatory cytokines, human beta-defensins (hBD) and markers like HLA-DR; this may lead to squamous metaplasia of conjunctival epithelial cells and reduction of goblet cell density $[8,9]$.

Most investigators now believe that the primary etiologic factors of the disease may contribute to the development of ocular lesions, since early conjunctival surface changes, tear film alterations, and meibomian gland dysfunction may be seen in patients with mild to

\section{Journal of Ocular Biology}

\author{
Rahul Bhargava ${ }^{1 *}$ and Prachi Kumar ${ }^{2}$
}

${ }^{1}$ Laser Eye Clinic, Noida 201301, India

${ }^{2}$ Department of Pathology, Santosh Medical College and Hospital, Ghaziabad 201301, India

*Address for Correspondence

Rahul Bhargava, Director, Laser Eye Clinic, B2/004, Ananda Apartments, Sector-48, Noida 201301, India, Tel: +919999055223; E-mail: brahul_2371@yahoo.co.in

Submission: 16 January 2015

Accepted: 30 January 2015

Published: 04 February 2015

Reviewed \& Approved by: Dr. Mark Willcox, Professor, School of Optometry and Vision Science, University of New South Wales, Sydney, Australia

moderate psoriasis. However, the efficacy of routine tear film tests like Schirmer, tear film break up time (TBUT) and Rose Bengal staining (RBS) for dry eye diagnosis in patients with psoriasis has not been defined. Secondly, the impact of psoriasis on ocular surface health and cytological changes in conjunctiva needs to be evaluated more extensively.

Ocular surface health may be assessed by conjunctival impression cytology (CIC), which allows cells to be harvested from ocular surface non-invasively; cells are removed by application of cellulose acetate filters or biopore membranes and subsequently analyzed by various methods depending on study objective or pathology involved. CIC can be used in conjunction with techniques like light microscopy, flow cytometry, RT-PCR amplification and immunohistochemistry, to aid in diagnosis and providing insight into mechanism and pathogenesis of dry eye disease [10].

The present study evaluated the efficacy of routine tear film tests and CIC for dry eye diagnosis in symptomatic patients with psoriasis as compared to age and sex matched healthy controls. The study also evaluated the correlation of these parameters with dry eye symptomatology and severity.

\section{Methods}

A case control study was performed at two referral eye centers in northern part of the Indian sub-continent. The trial was approved by the institutional review boards and the local ethics committee. A written informed consent was obtained from all patients willing to participate in the study based on Helsinki protocol.

\section{Inclusion criteria}

The diagnosis of psoriasis was made by a single dermatologist (SA) and the severity of skin disease assessed based on the Psoriasis Area and Severity Index (PASI) [11-14]. Symptomatic patients with psoriasis (chronic plaque/erythrodermic/pustular types) referred from dermatology or rheumatology clinics participated in the study. Subjects were recruited based on their response to (Dry Eye Scoring System, DESS $\odot$ ), a questionnaire of dry eye related symptoms (Table 1). All patients were also questioned for disease duration, joint involvement, and psoriasis treatment modalities received. Age and 
sex matched subjects without skin or ophthalmic disease, obtained from the same population/geographic area served as controls.

\section{Exclusion criteria}

Patients having current ocular infection, psoriatic uveitis or episcleritis, past history of herpetic eye disease, liver disease, diabetes and laser in situ keratomileusis were excluded from the study. Pregnant and lactating mothers, post-menopausal women, cognitive or psychiatric disorders, human immunodeficiency virus and hepatitis B or C infection, current use of punctual plugs, antiglaucoma drugs, oral anti-coagulants, topical corticosteroids (four to six weeks prior to study enrolment) and anti-cholinergics were also excluded. Patients allergic to fluorescein and malignancy or chronic infection of lacrimal gland were also excluded.

\section{Power calculation}

Power was calculated using DESS to detect clinical significance between cases and controls after data analysis, based on data from previously published study [15]. Taking a lead from this study, power was calculated using primary outcome measure (DESS) and assumptions were as follows: Odds ratio $=9.8$, exposed controls $=$ $29 \%$, one-sided alpha risk $=5 \%$, controls $/$ case ratio $=1.07$, total exposed $=48.3086 \%$, Estimated power $=99.9999 \%$.

\section{Ophthalmic examination and measurements}

The dry eye scoring system (DESS) ${ }^{\odot}$ was administered to all participants (cases well as controls) prior to ophthalmic examination and tests. A score was assigned to common symptoms of dry eye (Table 1). DESS is assessed on a scale of $0-18$, with higher scores representing dry eye severity. A symptom score of 0-6 represents mild, 6.1-12 moderate, and 12.1 to 18 , severe dry eye [16]. Following DESS questionnaire, the subjects had a complete ophthalmic examination done by an independent investigator (not a study surgeon) and included recording of corrected distance visual acuity (CDVA), slit-lamp examination; this included anterior chamber for cells and flare, assessment of lid margins, eye lashes, and meibomian gland orifice for any blockage or occlusion and dilated fundus examination with $+90 \mathrm{D}$ lens. One eye of each patient was selected at random for evaluation.

TBUT was first performed as eyelid manipulation may adversely influence the results. A sterile fluorescein strip containing $1 \mathrm{mg}$ fluorescein sodium (Madhu Instruments, Delhi, India) was applied over the inferior bulbar conjunctiva. The strip was moistened with normal saline solution prior to application. The patient was instructed to blink naturally, without squeezing, several times to distribute the

Table 1: Dry eye questionnaire and scoring system (DESS $\left.{ }^{\odot}\right)$.

\begin{tabular}{|l|c|c|c|c|}
\hline \multirow{3}{*}{ Symptom } & \multicolumn{4}{|c|}{ Score (maximum 18) } \\
\cline { 2 - 6 } & $\begin{array}{c}\text { Absent } \\
\text { (0) }\end{array}$ & $\begin{array}{c}\text { Some- } \\
\text { times (1) }\end{array}$ & $\begin{array}{c}\text { Frequent } \\
\text { (2) }\end{array}$ & Always present \\
\hline
\end{tabular}

Itching (or) burning

Sandy (or) gritty sensation

Redness

Blurring of vision

Ocular fatigue

Excessive blinking

aScores of 0 to 6 were mild, 6.1 to 12 were moderate, and 12.1 to 18 indicated severely symptomatic dry eye [12]. ( ) Bhargava R. Laser Eye Clinic, Noida, India fluorescein. The tear film was observed on slit lamp using cobalt blue filter. The interval between the last complete blink and the first appearance of a dry spot on cornea was recorded with a timer. Three readings were taken in succession and averaged. A tear film break up time of less than 10 seconds was considered diagnostic for dry eye [17].

The subject then waited for 30 minutes and Schirmer's test with anaesthesia was performed with eyes closed. The length of wetting on the filter paper after 5 minutes was recorded. Wetting less than $6 \mathrm{~mm}$ was considered diagnostic for dry eye [18].

After waiting for another 30 minutes, the Rose Bengal staining (RBS) was performed by applying a moistened sterile strip containing $1.5 \mathrm{mg}$ Rose Bengal over the inferior bulbar conjunctiva. A van Bjisterveld score of 4 or more was considered positive for dry eye syndrome [19].

Impression cytology technique: A single examiner performed CIC (by transfer method) and was masked to information obtained from the questionnaire [20]. The lacrimal lake at inner canthus was dried with a cotton tip applicator. A circular 0.22 micron filter paper measuring $13 \mathrm{~mm}$ in diameter (Sartorius, Gottingen, Germany) was grasped with a blunt tipped forceps and applied over the inferior bulbar conjunctiva. CIC samples were obtained from the non-exposed conjunctiva to eliminate the influence of environmental related factors on ocular surface in the exposed part. The paper strip was gently pressed with a glass rod held in the other hand. The filter paper was removed in a peeling fashion after 4-10 seconds and specimen transferred to the lab for fixation (ethyl alcohol, formaldehyde, and glacial acetic acid in 20:1:1volume ratio) and staining. Due to relative ease of handling, the filter paper was first placed on a glass slide with albumin paste to transfer the specimen to the slide, instead of working directly. However, loss of adhered material to the filter can be considered a potential disadvantage. The filter paper was then removed from the slide and the slide labelled and numbered. The slide was kept at room temperature and stained with periodic acid- Schiff and counterstained with hematoxylin and eosin. The mounted slide was first examined under the light microscope with 100x low power field (x 10 objective lens). After localization, cells were then analyzed with 400x final magnification (x 40 objective). At least 10 HPF were examined for goblet cells and epithelial cells. The number of goblet cells per HPF were marked and counted. Estimated GCD = number of goblet cells counted per HPF divided by sampling area covered in $\mathrm{mm}^{2}$. Grading and scoring was carried out by criteria suggested by Nelson [21].

Grade 0: The epithelial cells are small and round with eosinophilic staining cytoplasm. The nuclei are large, basophilic, with a nucleocytoplasmic ratio of 1:2. The goblet cells are abundant, plump and oval shaped having intensively PAS positive cytoplasm.

Grade I: The epithelial cells are slightly larger, more polygonal and have eosinophilic staining cytoplasm. The nuclei are smaller with a nucleo-cytoplasmic ratio of 1:3. The goblet cells are less in number, however they still maintain their plump oval shape, with an intensively PAS positive cytoplasm.

Grade II: The epithelial cells are larger and polygonal, occasionally multinucleated with variably staining cytoplasm. The nuclei are small 
with a nucleo-cytoplasmic ratio of 1:4 to 1:5. The goblet cells are markedly decreased in number and are smaller, less intensively PAS positive, with poorly defined cellular borders.

Grade III: The epithelial cells are large and polygonal with basophilic staining cytoplasm. The nuclei are small, pyknotic and in many cells, completely absent. The nucleo-cytoplasmic ratio is greater than 1:6. Goblet cells are completely absent. Nelson Grades 0 and 1 were regarded as normal, whereas grades 2 and 3 were considered to represent abnormal cytology.

\section{Statistics}

Statistical analysis was performed using SPSS software (version 22, IBM Inc.). Means of groups were compared using t tests. Chisquare tests were used for proportions. P value $<0.05$ was considered statistically significant. One way analysis of variance (ANOVA) was used when there were more than two groups (severity of dry eye disease and sub-type of psoriasis). Sensitivity and specificity of tear film tests for dry eye diagnosis in symptomatic patients was estimated by the area under receiver operating characteristic (ROC) curve. A correlation analysis (with regression) was done between dry eye symptoms and tear function tests in both groups, respectively. Adjusted Pearson's coefficient $\left(\mathrm{R}^{2}\right)$ and slopes of normal probability plots were compared; $R^{2}>0.5$ was considered significant.

\section{Results}

A total of 136 subjects were enrolled in the study. Eyes of 60 patients with psoriasis were compared to 76 eyes of age and sex matched controls after discarding 6 poorly stained CIC slides. Out of these, $46(76.7 \%)$ had chronic plaque type, 8 (13.3\%) had pustular and $6(10 \%)$ had erythrodermic psoriasis. The mean duration of skin disease was $6.9 \pm 4.6$ years. There was no significant difference in the severity of dry eye disease between the subtypes of psoriasis (ANOVA, $\mathrm{P}=0.456$ ).

The mean age was comparable amongst cases and controls $(\mathrm{P}=0.246)$. The difference in gender among cases and controls was not significant $(\mathrm{P}=0.445)$. There was a significant difference $(\mathrm{P}<0.001)$ in tear film parameters among cases and controls. Table 2 compares baseline characteristics of subjects in both groups, respectively.

At baseline, 3(5\%) cases were severely symptomatic, 36(60\%) moderately symptomatic, $13(21.6 \%)$ mildly symptomatic and 7(12\%) symptom free. Amongst controls, 2(2.6\%) were moderately symptomatic, $20(26.3 \%)$ mildly symptomatic and $54(71 \%)$ were symptom free $(\mathrm{P}<0.0001)$.

Out of 52(86.6\%) symptomatic psoriatic patients, Schirmer's test was positive in 20(33.3\%), 21 (35\%) had abnormal staining, 28(46.7\%) had TBUT consistent with dry eye and 21 (35\%) abnormal cytology (Nelson Grade 2 and 3); out of these, (17/28.3\%) patients had grade 2 changes (Figure 1).

Out of $22(28.9 \%)$ symptomatic controls, Schirmer's test was positive in 7 (9.2\%), 12(15.8\%) had abnormal staining, 11(14.6\%) had abnormal TBUT and none had abnormal cytology. In the control group, 68(89.5\%) hade grade 0 and $8(10.5 \%)$ had grade 1 changes.

The diagnostic accuracy of CIC, TBUT, Schirmer and RBS in psoriatic patients was evaluated and compared with age and sex matched controls. The area under the curve (AUC) was measured using ROC curve. The diagnostic accuracy was CIC (AUC=0.969) $>$ TBUT $($ AUC $=0.962)>$ Schirmer $($ AUC $=0.882)>$ RBS $($ AUC $=0.880)$ (Figure 2).

On correlation analysis (along with regression), there was a significant correlation between symptom severity and Nelson Grade (Pearson's correlation coefficient, $\mathrm{R}^{2}=0.88$ ). The correlation was not significant amongst controls $\left(\mathrm{R}^{2}=0.002\right)$ (Figure 3$)$. Likewise, TBUT and Schirmer also correlated well with symptom severity $\left(\mathrm{R}^{2}=0.630\right.$ and 0.612 , respectively) as compared to controls $\left(R^{2}=0.061\right.$ and 0.136 , respectively) (Figure 4). Moreover, there was a significant difference in the slope of the normal probability plots amongst cases and controls.

On simple linear regression, $84 \%$ variability in Nelson grade, $63 \%$ in TBUT, $61 \%$ in Schirmer and 33\% variability in RBS, respectively, could be explained by dry eye symptom severity. On analysis of variance, the probability corresponding to the $\mathrm{F}$ value $<0.0001$, suggests that there was less than $0.01 \%$ risk in assuming that the null hypothesis (no effect of dry eye symptoms) was wrong.

\section{Discussion}

The present study evaluated the role of routine tear function

Table 2: Baseline characteristics of patients.

\begin{tabular}{|c|c|c|c|}
\hline Parameter & Cases & Controls & t test (P value) \\
\hline Age (years) & $25.8 \pm 6.4$ & $24.9 \pm 5.6$ & 0.246 \\
\hline Symptom Score & $8.9 \pm 3.5$ & $1.1 \pm 1.6$ & $<0.001$ \\
\hline Schirmer (mm) & $12.8 \pm 4.8$ & $15.8 \pm 3.4$ & $<0.001$ \\
\hline TBUT (seconds) & $8.9 \pm 1.8$ & $12.8 \pm 1.9$ & $<0.001$ \\
\hline RBS & $2.3 \pm 1.5$ & $0.6 \pm 1.2$ & $<0.001$ \\
\hline Nelson Grade & $1.8 \pm 1$ & $0.7 \pm 0.5$ & $<0.001$ \\
\hline GCD (cells $/ \mathrm{mm}^{2}$ ) & $303 \pm 155$ & $699 \pm 137$ & $<0.001$ \\
\hline
\end{tabular}

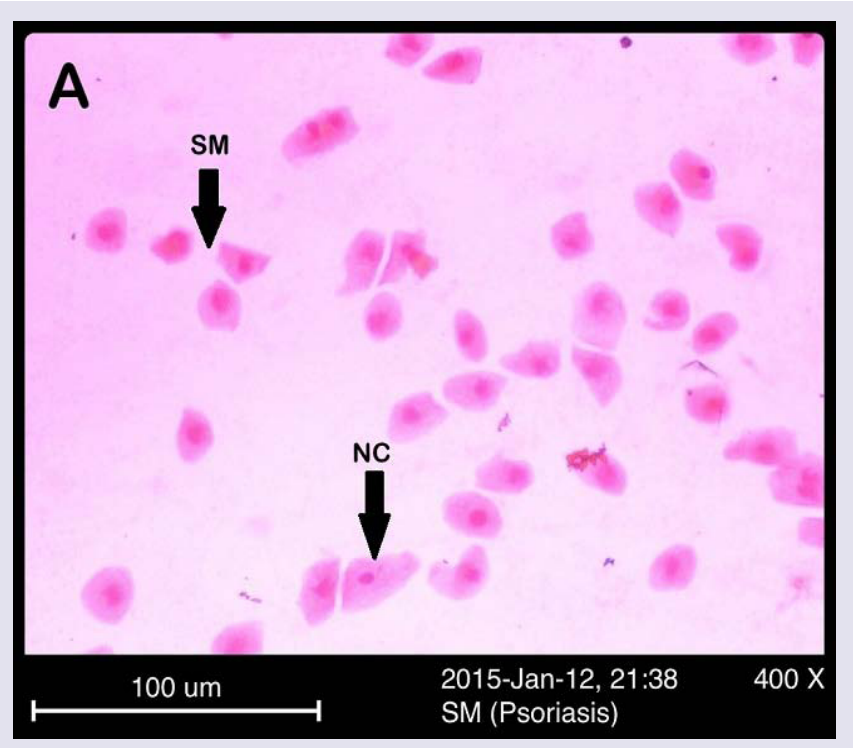

Figure 1: Photomicrographs of impression cytology specimens, stained with $\mathrm{PAS}$ and $\mathrm{H}-\mathrm{E}$ at $400 \mathrm{X}$ with squamous-metaplasia. Arrow showing a normal cell (NC) and increased nuclear-cytoplasmic ratio (SM), respectively. 

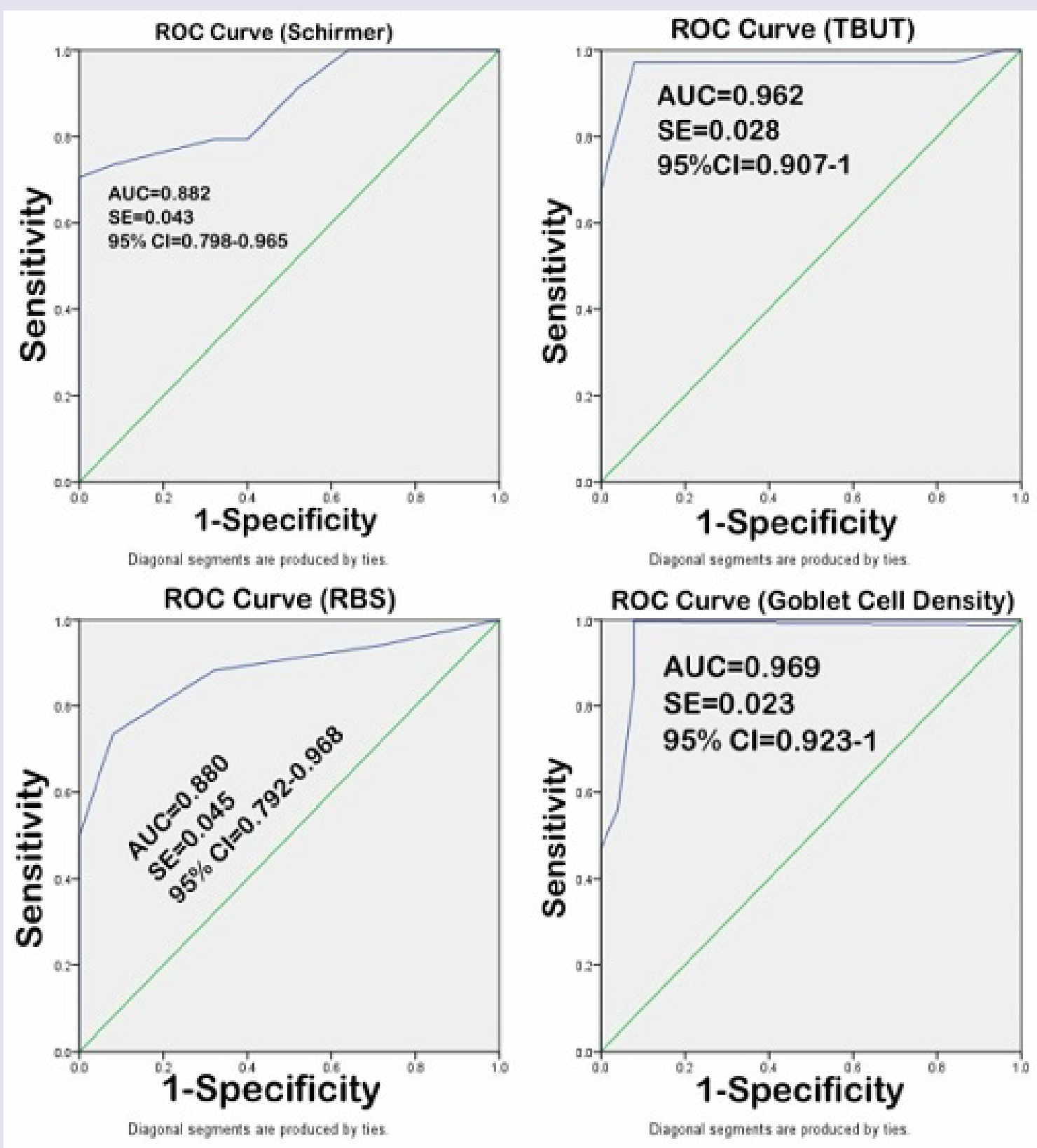

Figure 2: Receiver Operating Characteristic Curve (ROC) showing sensitivity and specificity of tear film tests.

tests and CIC for dry eye evaluation in patients with psoriasis. Dry eye diagnosis and its management has been a challenging task for ophthalmologists as most diagnostic tests for dry eye are poorly standardized, making compare between studies tenuous at best; a generally agreed upon 'gold standard' still does not exist [22].

Ocular signs and symptoms may be discordant; for instance, ocular symptoms might not occur despite reduction of tear production and not all symptomatic patients have abnormal tear function tests [23]. These were reinforced by the observation in the present study that among symptomatic psoriatic patients, $33.3 \%$ had abnormal Schirmer, $46.7 \%$ had tear film instability and $35 \%$ had altered cellular morphology. Secondly, 7(12\%) psoriatic patients, although asymptomatic at inclusion (according to DESS) had an abnormal
TBUT (<10 sec). Consequently, symptom based assessments have been a key component of clinical diagnosis in dry eye conditions; in dry eye research, a questionnaire can serve as a screening instrument and to define treatment groups according to symptoms [24].

In a case control study in 29 patients with psoriasis and 12 controls, Kumar et al found that dry eye disease was more severe (worse Nelson grade) in patients with pustular and erythrodermic psoriasis. However, the present study did not find any significant difference in disease severity or Nelson grade between subtypes of psoriasis (ANOVA, $\mathrm{P}=0.456$ ). Small sample size and different statistical method (Chi Square test versus ANOVA) could probably account for the difference in observation [25]. 
Normal P.P Plot of Regression Standardized Residual Dependent variable: Rose Bengal Staining

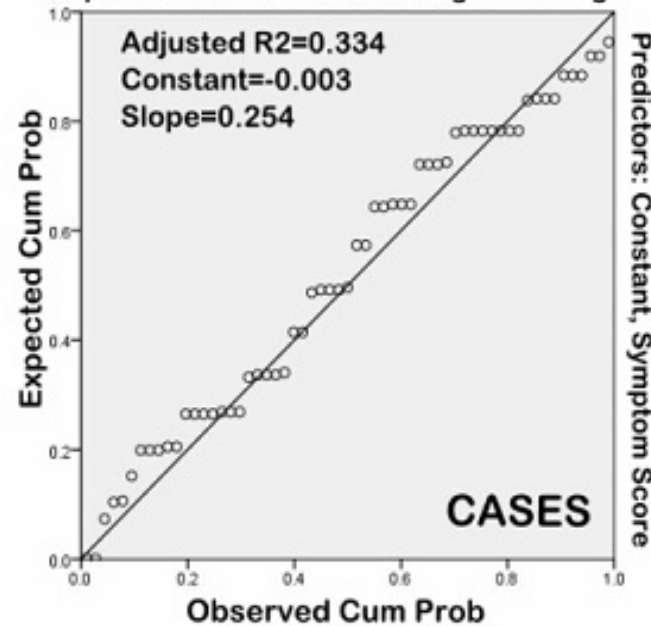

Normal P.P Plot of Regression Standardized Residual Dependent variable: Nelson Grade

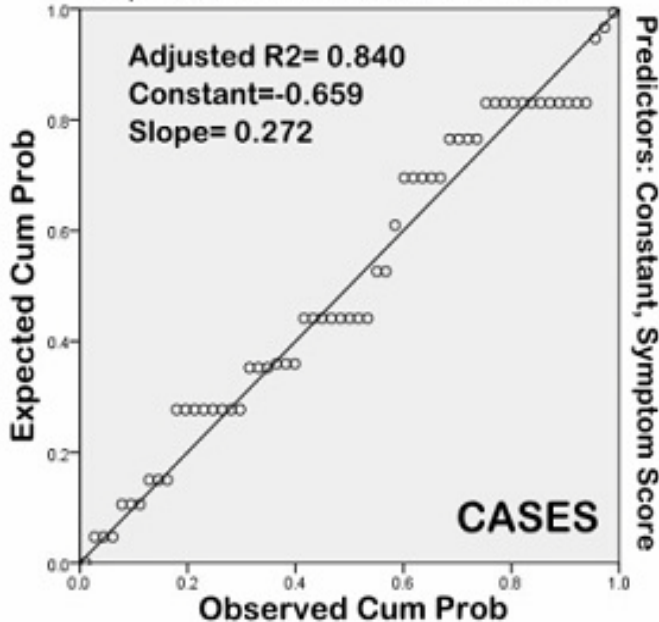

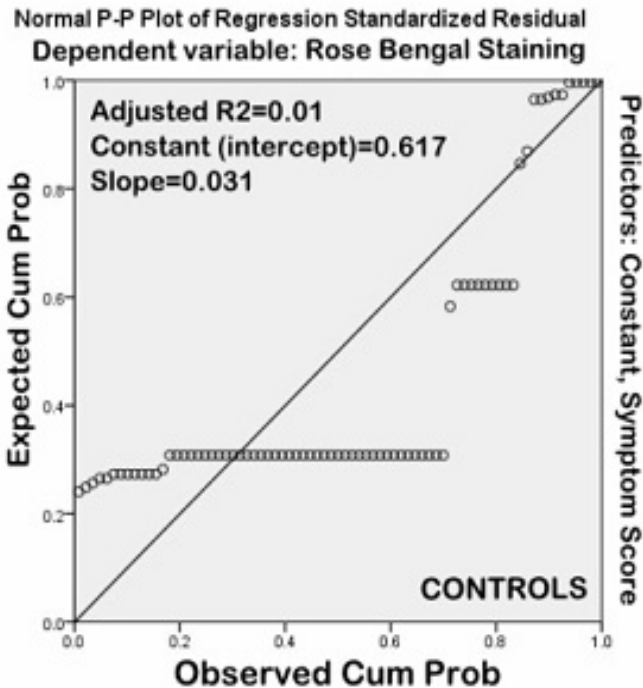

Normal P.P Plot of Regression Standardized Residual Dependent variable: Nelson Grade

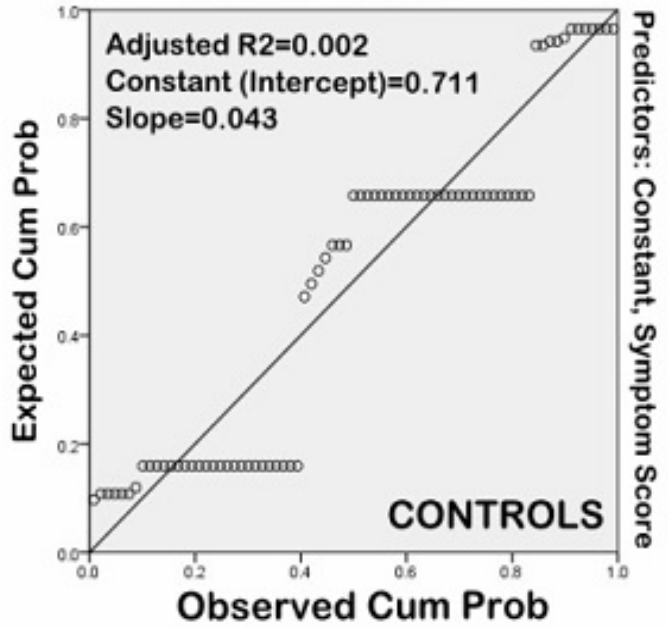

Figure 3: Normal probability plot of regression standardized residual; Dependent variable Rose Bengal Stain (RBS) and Nelson grade with symptom score.

In a case control study ( $\mathrm{n}=100$ eyes each), Karabulut et al. evaluated ocular-surface changes and tear-film functions in patients with plaque-type psoriasis. The authors found reduction in Schirmer and TBUT test values in symptomatic psoriatic patients; $20 \%$ patients had Nelson grade 2 changes on impression cytology as compared to $2 \%$ controls. An observation similar to the results of the present study [26].

In another study on 30 patients and equal number of healthy controls, Her et al. also found a significant reduction in TBUT, goblet cell density and alteration in epithelial cell morphology in cases as compared to controls. However, there was no difference in Schirmer test values between the groups. This finding was inconsistent with present and other studies in patients with psoriasis [27,28]. Small sample size of patients $(n=30)$ could probably explain for the difference in observation.

Bhargava et al. compared diagnostic accuracy of CIC, TBUT and
Schirmer test for dry eye evaluation in computer users in a large series of patients $(\mathrm{n}=715)$. Mean TBUT, Schirmer's test values, and goblet cell density were significantly reduced in computer users $(\mathrm{P}<0.001)$. TBUT, Schirmer's, and CIC were abnormal in $48.5 \%, 29.1 \%$, and $38.4 \%$ symptomatic computer users respectively as compared to $8 \%$, $6.7 \%$, and $7.3 \%$ symptomatic controls respectively. On correlation analysis, there was a significant correlation between dry eye symptoms, TBUT and CIC scores, in contrast to Schirmer's scores $\left(\mathrm{R}^{2}<0.5\right)$. The authors concluded that tear film tests should be used in combination with dry eye questionnaire for a more accurate dry eye evaluation. The present study found that CIC and DESS hold good diagnostic accuracy for dry eye evaluation in patients with psoriasis [15].

In a case control study on 216 eyes of patients with dry eye and 140 controls, Kumar et al found that GCD and TBUT were better predictors of morphological and cytological changes in the conjunctiva than Schirmer. Moreover, TBUT was more sensitive and specific than RBS and Schirmer for diagnosis of dry eye. An 
Normal P.P Plot of Regression Standardized Residual Dependent variable: Schirmer

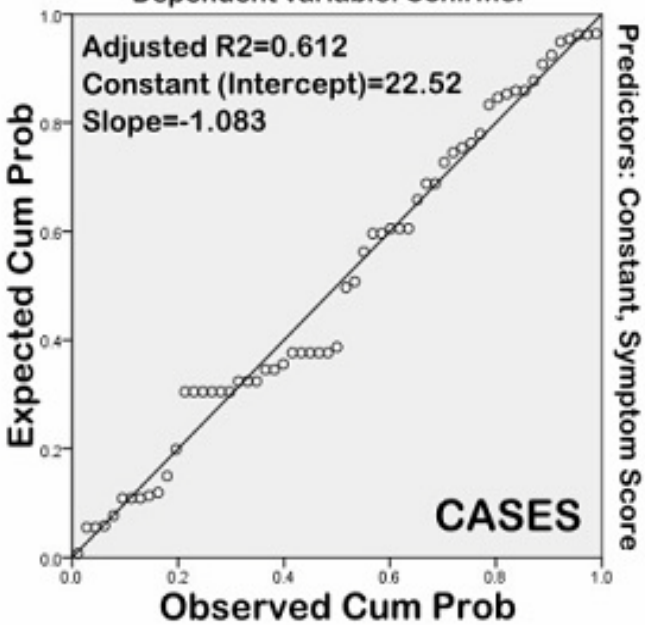

Normal P.P Plot of Regression Standardized Residual Dependent Variable: Tear Film Break Up Time

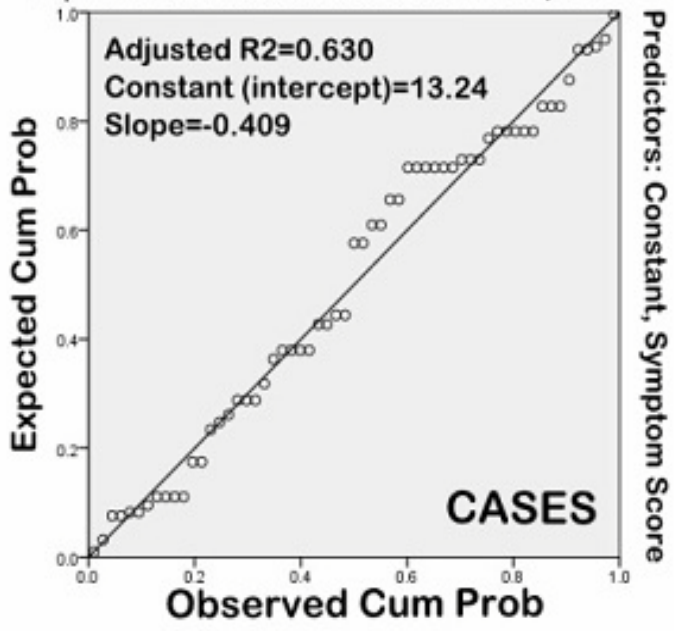

Normal P.P Plot of Regression Standardized Residual Dependent variable: Schirmer

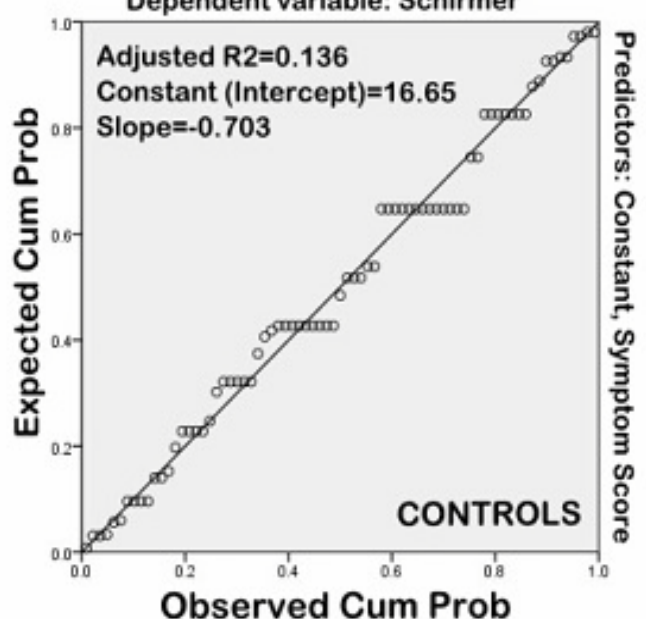

Normal P.P Plot of Regression Standardized Residual Dependent variable: Tear Film Break Up Time

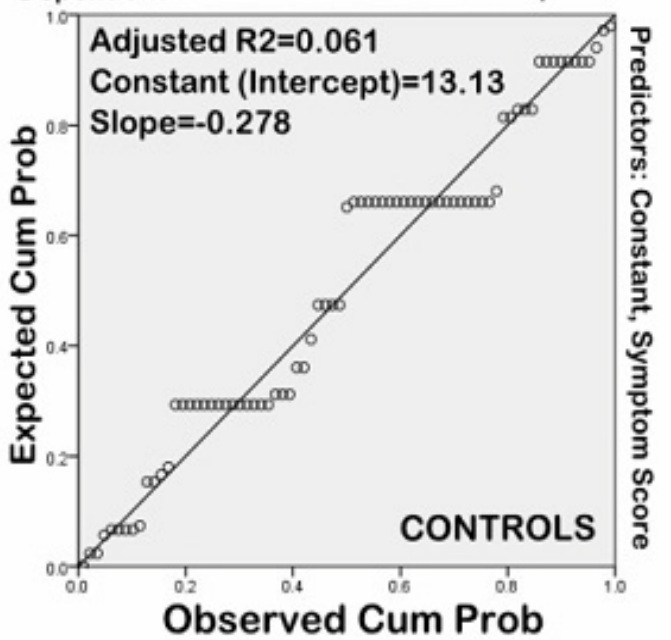

Figure 4: Normal probability plot of regression standardized residual; Dependent variable Schirmer and TBUT with symptom score.

observation, similar to the results of the present study [29].

In conclusion, Nelson grade correlates better with dry eye symptom severity as compared to TBUT, Schirmer and RBS. CIC is more sensitive and specific than routine tear function tests for dry eye evaluation in patients with psoriasis.

\section{References}

1. Augustin M, Krüger K, Radtke MA, Schwippl I, Reich K (2008) Disease severity, quality of life and health care in plaque-type psoriasis: a multicentre cross-sectional study in Germany. Dermatology 216: 366-372.

2. Lambert JR, Wright $V(1976)$ Eye inflammation in psoriatic arthritis. Ann Rheum Dis 35: 354-356.

3. Rehal B, Modjtahedi BS, Morse LS, Schwab IR, Maibach HI (2011) Ocular psoriasis. J Am Acad Dermatol 65: 1202-1212.

4. Kilic B, Dogan U, Parlak AH, Goksugur N, Polat M, et al. (2013) Ocular findings in patients with psoriasis. Int J Dermatol 52: 554-559.

5. Erbagci I, Erbagci Z, Gungor K, Bekir N (2003) Ocular anterior segment pathologies and tear film changes in patients with psoriasis vulgaris. Acta Med Okayama 57: 299-303.

6. Ayroldi E, Bastianelli A, Cannarile L, Petrillo MG, Delfino DV, et al. (2011) A pathogenetic approach to autoimmune skin disease therapy: psoriasis and biological drugs, unresolved issues, and future directions. Curr Pharm Des 17: 3176-3190.

7. (2007) The definition and classification of dry eye disease: report of the Definition and Classification Subcommittee of the International Dry Eye Workshop. Ocul Surf 5: 75-92.

8. Narayanan S, Miller WL, McDermott AM (2006) Conjunctival cytokine expression in symptomatic moderate dry eye subjects. Invest Ophthalmol Vis Sci 47: 2445-2450.

9. De Paiva CS, Villarreal AL, Corrales RM, Rahman HT, Chang VY, et al. (2007) Dry eye-induced conjunctival epithelial squamous metaplasia is modulated by interferon-gamma. Invest Ophthalmol Vis Sci 48: 2553-2560.

10. Demir HD, Aydın E, Sezer E, Yardım H (2013) Evaluation of plasma vitamin A and $E$ levels and tear film changes in patients with psoriasis vulgaris. Korean J Ophthalmol 27: 158-161. 
11. Yaylali V, Ozyurt C (2002) Comparison of tear function tests and impression cytology with the ocular findings in acne rosacea. Eur J Ophthalmol 12: 11 17.

12. Feldman SR, Krueger GG (2005) Psoriasis assessment tools in clinical trials. Ann Rheum Dis 64 Suppl 2: ii65-ii68.

13. Her Y, Lim JW, Han SH (2013) Dry eye and tear film functions in patients with psoriasis. Jpn J Ophthalmol 57: 341-346.

14. Campanati A, Neri P, Giuliodori K, Arapi I, Carbonari G, et al. (2014) Psoriasis beyond the skin surface: a pilot study on the ocular involvement. Int Ophthalmol [Epub ahead of print].

15. Bhargava R, Kumar P, Kaur A, Kumar M, Mishra A (2014) The diagnostic value and accuracy of conjunctival impression cytology, dry eye symptomatology, and routine tear function tests in computer users. J Lab Physicians 6: 102 108.

16. Bhargava R, Kumar P, Kumar M, Mehra N, Mishra A (2013) A randomized controlled trial of omega- 3 fatty acids in dry eye syndrome. Int $\mathrm{J}$ Ophthalmol 6: 811-816.

17. Pflugfelder SC, Tseng SC, Sanabria O, Kell H, Garcia CG, et al. (1998) Evaluation of subjective assessments and objective diagnostic tests for diagnosing tear-film disorders known to cause ocular irritation. Cornea 17: 38-56.

18. Savini G, Prabhawasat P, Kojima T, Grueterich M, Espana E, et al. (2008) The challenge of dry eye diagnosis. Clin Ophthalmol 2: 31-55.

19. van Bijsterveld OP (1969) Diagnostic tests in the Sicca syndrome. Arch Ophthalmol 82: 10-14.
20. Bhargava R, Kumar P (2014) Conjunctival impression cytology in computer users. Int J Ophthalmic Pathol 3: 4.

21. Nelson JD, Havener VR, Cameron JD (1983) Cellulose acetate impressions of the ocular surface. Dry eye states. Arch Ophthalmol 101: 1869-1872.

22. Kumar P, Bhargava R, Kumar M, Jyotsana M (2013) Dry eye syndrome: A diagnostic enigma. Int J Contemp Surg 1: 72-77.

23. Nichols KK, Nichols JJ, Mitchell GL (2004) The lack of association between signs and symptoms in patients with dry eye disease. Cornea 23: 762-770.

24. Finis D, Pischel N, König C, Hayajneh J, Borrelli M, et al. (2014) Comparison of the OSDI and SPEED questionnaires for the evaluation of dry eye disease in clinical routine. Ophthalmologe 111: 1050-1056.

25. Kumar P, Maurya RP, Bhushan P, Singh VP, Singh MK, et al. (2012) A study of conjunctival impression cytology in patients with various types of psoriasis. World J Pathol 1: 11.

26. Karabulut AA, Yalvac IS, Vahaboglu H, Nurozler AB, Duman S (1999) Conjunctival impression cytology and tear-film changes in patients with psoriasis. Cornea 18: 544-548.

27. Her Y, Lim JW, Han SH (2013) Dry eye and tear film functions in patients with psoriasis. Jpn J Ophthalmol 57: 341-346.

28. Gudmundsen KJ, O'Donnell BF, Powell FC (1992) Schirmer testing for dry eyes in patients with rosacea. J Am Acad Dermatol 26: 211-214.

29. Kumar P, Bhargava R, Kumar M, Ranjan S, Kumar M, et al. (2014) The correlation of routine tear function tests and conjunctival impression cytology in dry eye syndrome. Korean J Ophthalmol 28: 122-129. 